\title{
Rationalizing the Impact of Surface Depletion on Electrochemical Modulation of Plasmon Resonance Absorption in Metal Oxide Nanocrystals
}

Ankit Agrawal, ${ }^{\dagger} \| \odot$ Ilka Kriegel, ${ }^{\ddagger},, \| \odot$ Evan L. Runnerstrom, ${ }^{\dagger,}, \perp \odot$ Francesco Scotognella, Anna Llordes, ${ }^{\$ \subset}$ and Delia J. Milliron ${ }^{*}, \dagger, \S \odot$

${ }^{\dagger}$ McKetta Department of Chemical Engineering, The University of Texas at Austin, Austin, Texas 78712, United States

${ }^{\ddagger}$ Department of Nanochemistry, Istituto Italiano di Tecnologia (IIT), via Morego, 30, 16163 Genova, Italy

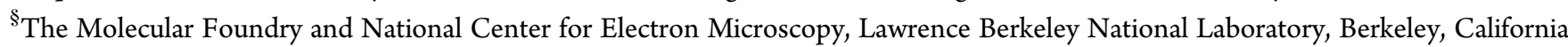
94720, United States

${ }^{\perp}$ Department of Materials Science and Engineering, University of California-Berkeley, Berkeley, California 94720, United States

\# Dipartimento di Fisica, Politecnico di Milano, Piazza Leonardo da Vinci 32, 20133 Milano, Italy

\section{Supporting Information}

ABSTRACT: Dynamic control over the localized surface plasmon resonance (LSPR) makes doped metal oxide nanocrystals (NCs) promising for several optoelectronic applications including electrochromic smart windows and redox sensing. Metal oxide NCs such as tin-doped indium oxide display tunable infrared LSPRs via electrochemical charge injection and extraction as a function of the externally applied potential. In this work we have employed dispersion phase electrochemical charging/discharging to study the mechanism behind the optical modulation on an individual NC scale. The optical modulation of the LSPR is dominated by a sharp variation in intensity during reduction and oxidation along with an only modest shift in the LSPR frequency. With a core-shell modeling approach, in which an active NC core surrounded by a depleted shell is assumed, we were able to reproduce the trends in and main features of our experimental results. The shell thickness depends on the applied potential and we extracted the temporal evolution of the shell thickness together with the variation of the Drude parameters until equilibrium was reached. The variation of the core versus shell volume fraction as a function of electrochemical potential reinforces the importance of the depletion layer in highly doped NCs and uncovers important implications on their near and far field plasmonic properties.

KEYWORDS: LSPR, near-field enhancement, metal oxide, tunable plasmonics, depletion
T $\mathrm{n}$ degenerately doped semiconductor nanocrystals (NCs) the localized surface plasmon resonances (LSPR), originating from the collective oscillations of the free electrons in the conduction band, are centered in the infrared spectrum., Depending on the dopant type, dopant concentration, shape, and size of the NCs, the LSPR can be tuned across the nearinfrared (NIR) to the far-infrared spectral window. ${ }^{1,3-5}$ Notably, LSPRs in semiconductor NCs can also be dynamically modulated via external stimuli, such as chemical, photochemical, or electrochemical doping. ${ }^{6-8}$ In particular, the control of the LSPR via an external applied potential has shown great promise for electrochromic smart window operation. ${ }^{6,9}$ Metal oxide nanocrystals such as $\mathrm{WO}_{3-x}$ and $\mathrm{Sn}: \mathrm{In}_{2} \mathrm{O}_{3}$ (ITO) are key components in smart windows that in complex nanostructured ensembles enable the independent tuning of visible and NIR light transmission, fast switching times, and high optical modulation. ${ }^{6,9-11}$ Until recently, the focus of most of the research went toward the optimization of optical and electrochemical performance via material design and structural engineering, but little knowledge has been acquired on the actual mechanism behind the electrochemical switching. ${ }^{12}$ In our recent work, ${ }^{13}$ we found that the optical properties of doped metal oxide NCs are largely influenced by the presence of a depletion region, resulting from NC surface states, that reaches into the NC volume with radial dimension from subnanometer $(\mathrm{nm})$ to several nanometers in thickness. This translates to a volume fraction of up to $80 \%$ of the entire NC volume (for low doped sub-5 nm diameter NCs), thus, greatly perturbing the LSPR properties of the NCs. ${ }^{13} \mathrm{We}$ moreover found that by controlling the equilibrium Fermi level under an applied external potential, the thickness and carrier

Received: December 21, 2017

Published: March 21, 2018 

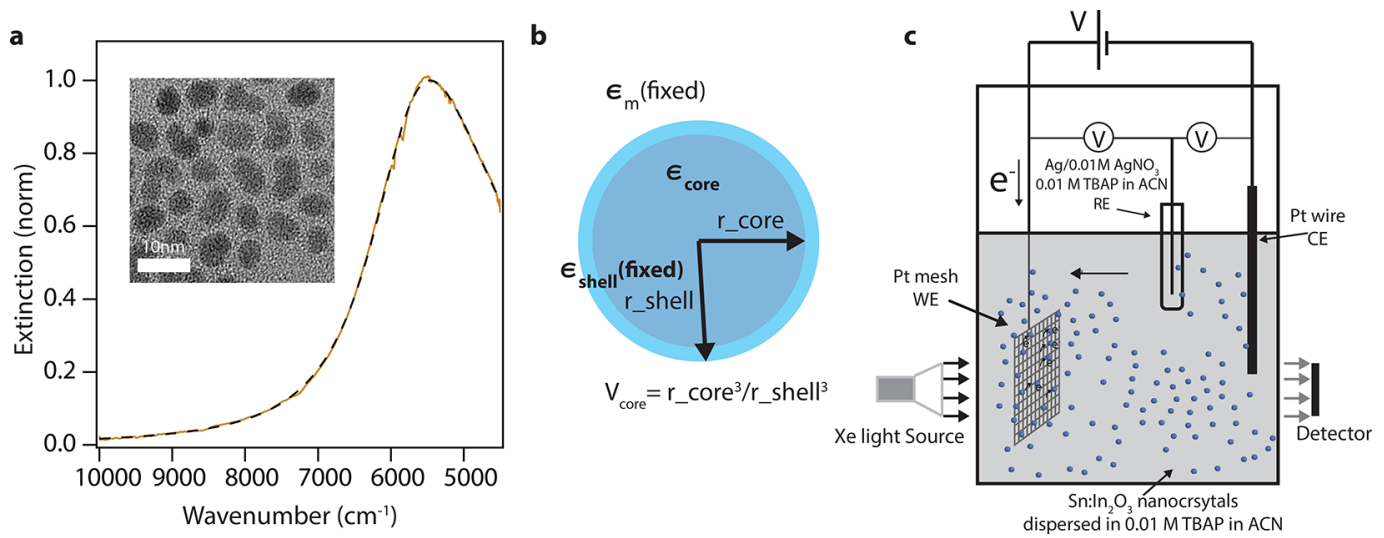

Figure 1. (a) Typical absorbance spectrum of the $\mathrm{Sn}: \mathrm{In}_{2} \mathrm{O}_{3}$ NCs in a $0.01 \mathrm{M}$ TBAP in ACN solution (black dashed curve) with a fit according to the Drude model and the EMA (yellow curve). Inset: Typical TEM image of the $\mathrm{Sn}: \mathrm{In}_{2} \mathrm{O}_{3}$ NCs. (b) Sketch of the model used for the fit procedure. In dark blue is given the active core with radius $\left(r_{\text {core }}\right)$ and the frequency dependent dielectric function $\varepsilon_{\text {core }}$. The light blue area surrounding the core depicts the depleted shell with a constant dielectric constant $\left(\varepsilon_{\text {shell }}\right)$, all immersed in a solvent with dielectric constant $\varepsilon_{\mathrm{m}}$. The volume fraction is then given as $V_{\text {core }}=r_{\text {core }}^{3} / r_{\text {shell }}^{3}$. (c) Sketch of the spectroelectrochemical setup composed of a cuvette with $1 \mathrm{~mm}$ path length, a Pt-mesh working electrode and a nonaqueous $\mathrm{Ag} / \mathrm{Ag}^{+}$reference electrode (not to scale).

concentration within this depletion layer can be modulated, thereby affecting the NCs' LSPR properties, and that the extent of LSPR shift and absorption intensity modulation strongly depends on the NC size and the dopant concentration inside the host lattice.

Recently, modulation of LSPR in NC films was analyzed in the context of depletion effects impacting their optical properties. ${ }^{13,14}$ In that case, the LSPR is broadened and redshifted due to near-field interactions between neighboring NCs. Additionally, there is substantial background contribution to the signal arising from the substrate and the underlying conductive layer. Both of these effects make it difficult to study the fundamental charging and discharging process in individual nanocrystals. $^{15-19}$ Therefore, to investigate the effects of surface depletion on the modulated LSPR of individual, isolated nanocrystals, we use solution electrochemistry to assess the optical modulation of $\mathrm{Sn}: \mathrm{In}_{2} \mathrm{O}_{3} \mathrm{NC}$ LSPR spectra, thus, getting closer to single particle measurements. ${ }^{18,20-25}$ In this way, we were able to examine LSPR modulation in NC dispersions where $\mathrm{NC}-\mathrm{NC}$ interaction is absent and this enabled us to make quantitative comparisons between values extracted by fitting our experimental results and our theoretical model.

Here, we measure electrochemical charge injection and extraction in $10 \%$ Sn-doped $\operatorname{In}_{2} \mathrm{O}_{3}$ NCs dispersed in acetonitrile using a spectroelectrochemical apparatus composed of a platinum mesh working electrode and a $\mathrm{Ag} / \mathrm{Ag}^{+}$reference electrode submerged in the NC dispersion. We fit the resulting spectral variations using a core-shell effective medium approach, assuming that the NC consists of a plasmonically active $\mathrm{Sn}: \operatorname{In}_{2} \mathrm{O}_{3}$ core surrounded by a uniformly depleted shell of the same material, in accordance with our recent findings. ${ }^{13}$ With this model we quantify the underlying processes of charging and discharging the $\mathrm{Sn}: \mathrm{In}_{2} \mathrm{O}_{3} \mathrm{NCs}$ by assessing the relative spectral contributions of charge carrier density, carrier damping and depletion region together in our electrochemically tuned plasmonic system. We find that depletion layer thickness plays a fundamental role and is in fact primarily responsible for the uptake of extra charges in the reduction process, thus reducing the depletion thickness, while charge extraction or oxidation results in a further inward growth of depletion layer. Consequently, we find that the total carrier density is not significantly altered at any point during the charging and discharging process. This is of fundamental importance for the plasmonic response of electrochemically modified NCs, as the depletion layer largely dominates not only the intensity and peak position of the LSPR, but also is expected to substantially impact the near-field LSPR properties. Thus, we unravel not only the underlying nanoscale effects occurring during the electrochemical charging process, but also highlight implications of this effect on the optical response of the modulated plasmonic material.

\section{RESULTS AND DISCUSSION}

$\mathrm{Sn}: \mathrm{In}_{2} \mathrm{O}_{3}$ NCs were synthesized using air-free colloidal synthesis techniques by the decomposition of metal oleates in organic solvent ${ }^{26-28}$ (see Supporting Information for synthesis details). The optical extinction spectrum (Figure 1a) of a representative sample, dispersed as isolated NCs, displays an intense LSPR centered at $5494 \mathrm{~cm}^{-1}$. A typical transmission electron microscopy (TEM) image (Figure la, inset) illustrates the pseudospherical $\mathrm{Sn}: \operatorname{In}_{2} \mathrm{O}_{3} \mathrm{NCs}$ with an average diameter of around $5 \mathrm{~nm}$. We modeled the LSPR spectrum using a coreshell effective medium model to derive the electronic properties such as free carrier concentration and electron scattering (Figure 1b, see Supporting Information for more detail). The free carrier concentration in the initial state, extracted using core-shell model, was $1.28 \times 10^{21} \mathrm{~cm}^{-3}$. Previous LSPR modulation studies completed on $\mathrm{NC}$ films suggest that at these high carrier concentrations, strong NC-NC coupling and small depletion width (less than $1 \mathrm{~nm}$ ) result in small LSPR frequency modulation upon applied electrochemical bias. ${ }^{12,13}$ To mitigate the effects of NC-NC coupling and study depletion layer modulation directly, we used these $\mathrm{Sn}: \mathrm{In}_{2} \mathrm{O}_{3}$ $\mathrm{NCs}$ as a model system for solution electrochemical modulation experiments, as illustrated in Figure 1c.

The LSPR of our NCs was tuned in a spectroelectrochemical apparatus composed of a platinum mesh as a working electrode, a $\mathrm{Ag} / \mathrm{Ag}^{+}$reference electrode, and a Pt wire counter electrode. The counter electrode was a thin Pt wire, not isolated from the nanocrystal solution. However, the low surface area and applied potential range avoided any opposite reduction or oxidation reaction to take place at the counter electrode. The solution was not stirred due to the very small sample compartment, so 
that the reactions occurred were limited by diffusion. A sketch of the experimental setup can be found in Figure 1c and in the SI. To simultaneously facilitate charge transfer into and out of individual nanocrystals, while maintaining a stable colloidal dispersion within the electrolyte, we chose very small NCs of about $5 \mathrm{~nm}$ in diameter. Moreover, the native oleate ligands were stripped and the NCs were subsequently wrapped with polyethylene glycol (see SI for additional details). The polymer-wrapped $\mathrm{Sn}: \mathrm{In}_{2} \mathrm{O}_{3} \mathrm{NCs}$ were then easily dispersed in a $0.01 \mathrm{M}$ tetrabutyl ammonium perchlorate (TBAP) acetonitrile electrolyte solution (Figure 1c). Spectroelectrochemical measurements were performed in a quartz cuvette with an optical path length of $1.0 \mathrm{~mm}$. The open circuit potential (OCV) of the electrochemical setup was $0.2 \mathrm{~V}$ versus the reference electrode and the electrochemical reduction and oxidation reactions were triggered by applying a constant -1.5 and $1 \mathrm{~V}$, respectively, representing the limiting values given the chosen electrochemical system, that is, maximum reducing and oxidizing potentials. The time-dependent LSPR spectra were recorded during electrochemical charging and discharging. The electrochemical charging process was terminated when both the LSPR peak position and LSPR intensity saturated to a particular value or after a certain period of time. A time range of approximately $2 \mathrm{~h}$ has proven sufficient to reach saturation and avoided termination due to evaporation of the solvent.

Under reducing conditions, the LSPR spectrum shifts toward higher energies (blue-shift), accompanied by an increase in absorbance. In the reverse condition upon oxidation, an LSPR shift toward lower energies (red-shift) is induced together with a decrease in intensity. We hypothesize that during charging or discharging, as a result of random Brownian motion of the NC, whenever a NC hits the working electrode, an electron transfer process takes place that leads to the change in the NC LSPR properties. $^{22,29,30}$ As the charging and discharging of the NCs relies on diffusion, analogous to a Faradaic charge transfer reaction, when starting from the $\mathrm{OCV}$ condition it requires substantial time to reach the equilibrium reduced or oxidative state. A charging and discharging process took place for approximately $60 \mathrm{~min}$ during oxidation and reductive potential sweep, respectively. Notably, the charging and discharging process is reversible: The initial blue-shift and increase in LSPR intensity (Figure 2a, red) under reducing conditions $(-1.5 \mathrm{~V}$ ) is completely reversed on application of an oxidizing potential $(1 \mathrm{~V})$, exceeding the initial OCV LSPR (Figure 2a, black) to end up at a red-shifted and lower intensity LSPR than initially observed (Figure 2a, blue). Similar results are observed in the reverse configuration, when a positive potential is initially applied (Figure 2b). These experiments indicate that the injected electrons responsible for the blue-shift during reduction are completely removed upon oxidation, with additional extraction of some free carriers introduced into the $\mathrm{NC}$ by Sn doping. Moreover, the approximate reaction times required to introduce and remove carriers from the initial $\mathrm{Sn}: \mathrm{In}_{2} \mathrm{O}_{3} \mathrm{NCs}$ are evidently different. In five continuous cycles we switched between $1 \mathrm{~V}$ for oxidation and $-1.5 \mathrm{~V}$ for reduction and tracked the spectra over time (Figure $2 \mathrm{c}, \mathrm{d}$ ). The reversibility of injection and extraction of carriers is consistent over several cycles. We found it necessary to allow twice the time for the oxidation step to complete the charge extraction. Despite allowing extra time, the intensity and LSPR shift upon oxidation was significantly lower than for reduction. As a consequence, after five cycles a red shift beyond the initial
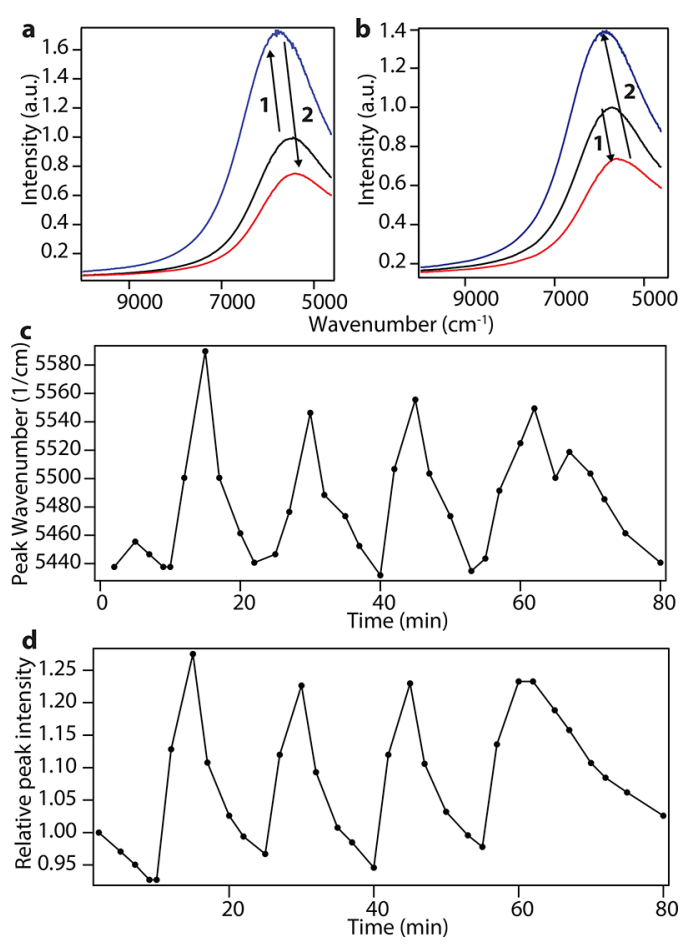

Figure 2. (a) The process of reduction and oxidation in a sequential manner: the initial blue-shift and increase in intensity, when applying a negative potential (black to blue curve), is completely reversed when switching to a positive potential, finally surpassing to a red-shifted and intensity decreased LSPR (red spectrum). (b) The process of oxidation and reduction in a sequential manner: the initial red-shift and decrease in intensity, when applying a positive potential (black to red curve) is completely reversed when switching to a negative potential, exceeding to a blue-shifted and intensity increased LSPR (blue spectrum). (c) Summary of the change in LSPR peak wavenumber vs time for five cycles. (d) Summary of the change in peak intensity over time for five cycles.

position was not achieved. These results indicate that charge injection is faster kinetically and per electron injected induces larger optical change both in intensity and LSPR energy.

To investigate the underlying mechanism behind the LSPR modulation upon electrochemical oxidation and reduction, the optical extinction was collected until optical steady state was reached. The extinction spectra were collected in time intervals of $1 \mathrm{~min}$ over a total time of approximately $2 \mathrm{~h}$ for the reduction (Figure $3 \mathrm{a}$ ) and about $1 \mathrm{~h}$ for the oxidation (Figure $3 b)$. At fixed $-1.5 \mathrm{~V}$ potential, during reduction, the LSPR spectrum shifted toward higher energies from 5461 to 5896 $\mathrm{cm}^{-1}$, accompanied by an increase in absorbance by a factor of 2.48. In the reverse condition, upon oxidation at constant $1 \mathrm{~V}$ potential, an LSPR shift toward lower energies from 5555 to $5482 \mathrm{~cm}^{-1}$ is induced together with a decrease in intensity by a factor of 0.80 . Previously, the modulation of the LSPR has been assigned largely to a changing carrier density using the framework of the Drude model and Mie theory. ${ }^{7,31}$ However, this simple picture cannot explain the observed significant changes in absorption intensity during electrochemical modulation with the only minor accompanying variation in LSPR peak energy. Also, the change in the surrounding refractive index of the medium, for example, due to a variation of the relative concentration of different ionic species around the charged NC has been excluded as a major source for the observed LSPR peak shifts. ${ }^{32,33}$ In a study by Brown et al. ${ }^{33}$ 
a

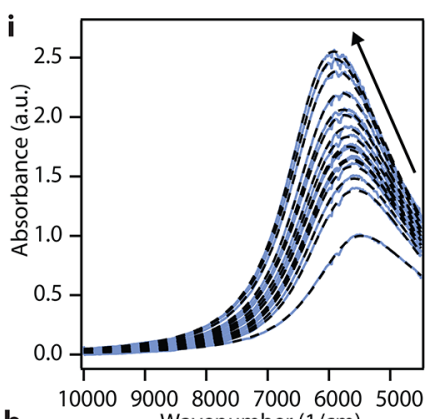

b

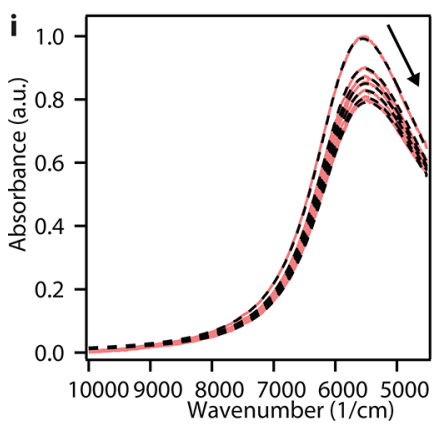

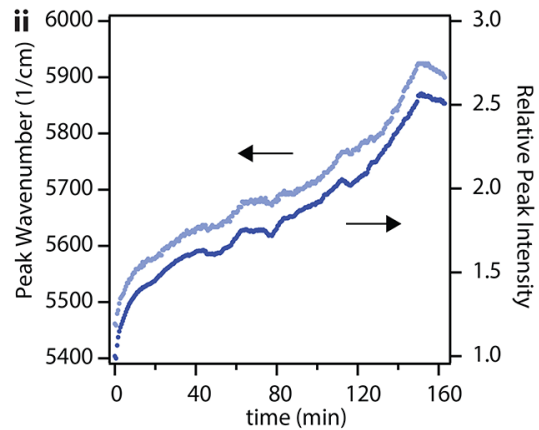
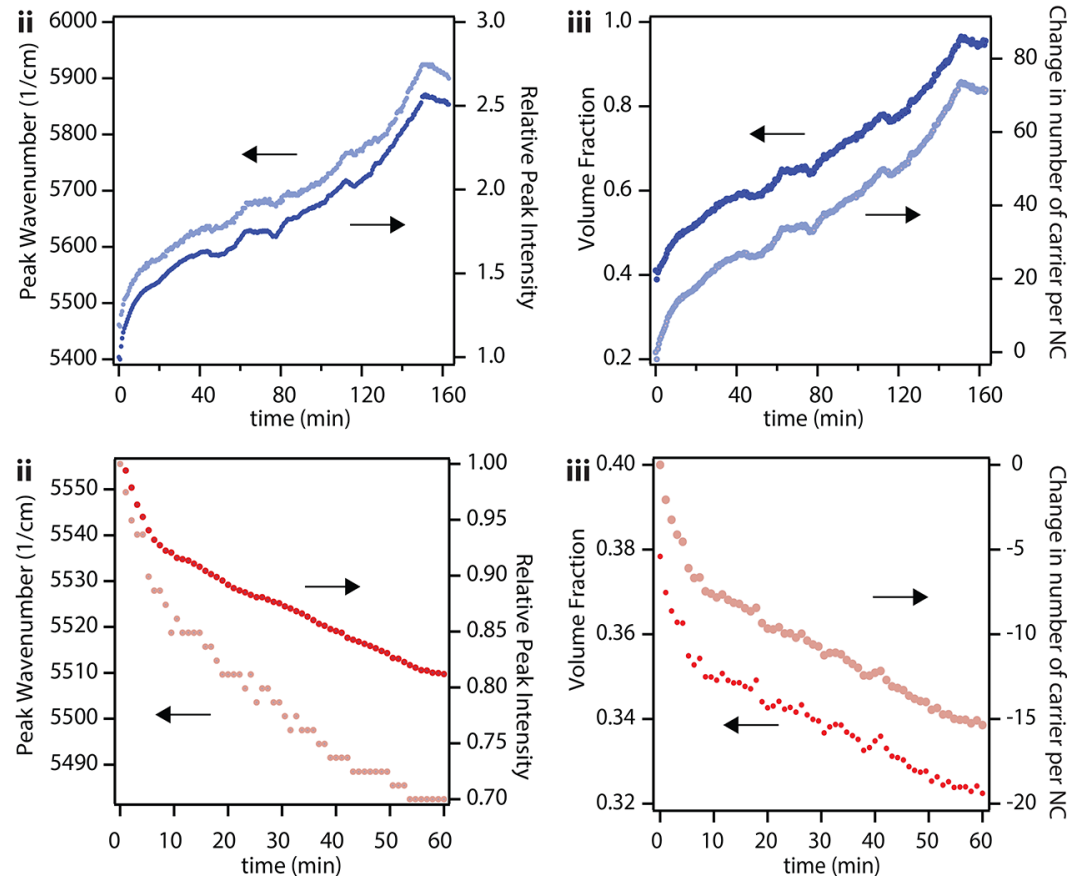

Figure 3. $(\mathrm{a}, \mathrm{i}$ and $\mathrm{b}, \mathrm{i})$ The results of a typical reduction/oxidation experiment $(-1.5 \mathrm{~V} / 1 \mathrm{~V})$, blue/red curves, respectively, for time intervals of approximately $1 \mathrm{~min}$ over a total time of $2 \mathrm{~h} / 1 \mathrm{~h}$. Black dashed curves represent the fit. (a,ii and b,ii) Plot of the relative peak intensity (blue/red curve) and LSPR wavelength vs time (light blue/light red curve) for the same experiments as in a,i and b,i. (a,iii and b,iii) Parameters extracted by fitting our model to the results. Blue/red curves give the change in volume fraction with reaction time, and light blue/light red curves depict the change in number of carriers injected/extracted from the nanocrystal.

where the electrochemical doping of gold nanoparticles was studied, a variation of the refractive index in the range of 0.004 was identified. A variation in this range, however, cannot explain the large changes in the LSPR we observe. In fact, we recently observed that for highly doped metal oxide NC films, it is the change in surface depletion thickness that results in LSPR modulation. ${ }^{13}$ To quantify the change in surface depletion thickness during electrochemical oxidation and reduction, we fitted the LSPR spectra with a core-shell optical model (for more fitting procedure details, see Supporting Information), in which we assumed an LSPR-active $\mathrm{Sn}: \mathrm{In}_{2} \mathrm{O}_{3}$ core surrounded by a uniform depleted shell made of the same material, but with a far lower electron contribution, and the entire $\mathrm{NC}$ is embedded in a surrounding medium. In Figure $1 \mathrm{~b}$ we show a sketch of the model used for the fit procedure, where the active $\mathrm{Sn}: \operatorname{In}_{2} \mathrm{O}_{3}$ core is given in dark blue with radius $\left(r_{\text {core }}\right)$ and the frequency dependent dielectric function $\varepsilon_{\text {core }}$. The light blue area surrounding the core depicts the depleted shell with shell dielectric constant $\left(\varepsilon_{\text {shell }}\right)$, all immersed in a solvent with dielectric constant $\varepsilon_{\mathrm{m}}$. The volume fraction is then given as $V_{\text {core }}$ $=r_{\text {core }}{ }^{3} / r_{\text {shell }}{ }^{3}$.

From the fits to the absorbance spectra of dilute dispersions of $\mathrm{Sn}: \mathrm{In}_{2} \mathrm{O}_{3}$ NCs we extracted the Drude parameters, namely the plasma frequency $\omega_{\mathrm{P}}$, the damping constants $\gamma(\omega)$, as well as the volume fraction of the LSPR active NC core $\left(V_{\text {core }}\right.$ Figure $1 \mathrm{~b}$ ). The volume fraction is a measure of the ratio between active core volume and inactive shell thickness and, thus, a measure for the variation of the depletion layer thickness: values approaching one indicate a decreasing depletion layer thickness, while smaller values indicate a larger volume of the $\mathrm{NC}$ consumed by the depletion region. In Figure la the spectra (yellow curve) along with the fit (black dashed curve) based on Mie theory is given and reveals an excellent overlap with the experiment. We extract a free carrier concentration of $1.28 \times 10^{21} \mathrm{~cm}^{-3}$ from this fit. A similar fitting procedure was employed to extract the time-dependent Drude parameters and changing $V_{\text {core }}$ during reduction and oxidation. We initialized each fit by using the fit parameters of the previous time as the new starting parameters for the next spectrum. The results of the fitting (Figure 3a,iii and b,iii) suggested that the NC core volume fraction increases as a function of time under reducing conditions (Figure 3a,iii) and decreases as a function of time under oxidative conditions (Figure 3b,iii). This change in the thickness of the depleted shell region effectively modulates the LSPR arising from the plasmonically active NC core. Under reducing conditions the high refractive index shell thickness is decreasing, leading to a slight shift of the experimental LSPR peak toward higher energy. But more importantly, the LSPR active core volume increases from $40 \%$ to $80 \%$. This large increase in the active core volume dramatically increases LSPR absorption intensity (Figure 3a, i and ii). The reverse effect under oxidative conditions leads to a decrease in LSPR active NC core volume from $40 \%$ to $32 \%$ resulting in a decrease in LSPR absorption intensity. The increasing thickness of the high-refractive index shell concomitantly leads to spectra shifting slightly to lower energy (Figure 3b). Furthermore, we could extract the number of free carriers injected into or extracted from the NCs using the plasma frequency and the active core volume determined by fitting. We found that approximately 84 electrons were injected into each NC during reduction, while only about 20 carriers were extracted relative to the initial state, explaining the difference in the extent of modulation during reduction and oxidation. The extracted plasma frequency value across both reduction and oxidation processes changed slightly in the range of $16400-16800 \mathrm{~cm}^{-1}$ (Figure S2). With only this slight 
change in plasma frequency and damping, the core-shell model was able to fit all of LSPR spectra during oxidation and reduction with high degree of robustness, with the active volume fraction being the most substantially modified physical parameter. This result reinforces the concept of depletion modulation being the primary mechanism behind the LSPR modulation in semiconductor NCs, especially at high doping levels. Furthermore, the presence of a high initial number of free carriers in our NCs excludes any significant contribution due to quantum effects, thus supporting the validity of conclusions reached by using continuum modeling in this work.

To test the validity of the core-shell model to predict the far field optical response and study the change in near-field intensity around the NCs during electrochemical modulation, we performed full field optical simulations using steady state carrier concentration profiles obtained by solving Poisson's equation over a spherical NC. ${ }^{34}$ Since the NCs are dispersed, we modeled isolated NCs to account for the optical response-a considerable simplification compared to earlier studies of NC thin films. ${ }^{13}$ The conduction band profile (Figure 4a,i) inside a NC was solved for three different equilibrium chemical potentials: (1) at $\mathrm{OCV}$, where the equilibrium potential is determined by the NC surface states, here likely surface hydroxyls. A high density of states due to this surface chemistry was assumed to occur at $0.35 \mathrm{eV}$ below the flat band conduction band minima. The hydroxyl surface states have been reported to pin the Fermi level near such energy level; ${ }^{25}$ (2) at a reducing potential $0.75 \mathrm{eV}$ above the surface state Fermi potential; as the NCs are reduced the Fermi level shifts above the OCV Fermi level; and (3) at oxidizing potential 0.75 $\mathrm{eV}$ below the surface state Fermi potential; as we oxidize the NCs the Fermi level shifts below the OCV Fermi level. The NC surface potential is directly proportional to the applied electrochemical potential, but as we do not know the exact correlation between the potentials, these reducing and oxidizing surface potentials were chosen as good estimates to simulate the trend in carrier concentration. The simulated band profiles suggest that the NCs are in the depleted state under OCV with the depleted shell accounting for $49 \%$ of NC volume, which is consistent with our volume fraction extracted from the coreshell model (Figure 4a,ii). Under reducing conditions, the externally added electrons diminish the depleted region to $11 \%$ of the NC volume; while under oxidative conditions, the removal of electrons extends the depletion region toward the core of the NCs, increasing the depleted NC volume to $66 \%$. Thus, in all three situations the NC can be approximated as having an LSPR active core and a depleted shell that varies in time until equilibrium is reached with an externally applied potential. The core-shell model fully captures the response of the LSPR to variable external potential, further validating its applicability to rationalize far field and near-field optical response.

Furthermore, to correlate the change in depletion region and LSPR modulation, we simulated (with COMSOL wave optics module) the far- and near-field LSPR properties of our NCs using the actual carrier concentration obtained through Poisson's equation. By using the radial distance-dependent carrier density profiles, we simulated the far-field LSPR spectra (Figure $4 \mathrm{~b}$ ) of the spherical NC and obtained remarkable consistency with the experimental observations. During the reduction process, the slight blue shift of the LSPR is well represented as well as the intense change in the magnitude of absorption. Similarly, the oxidized structure shows a slightly a
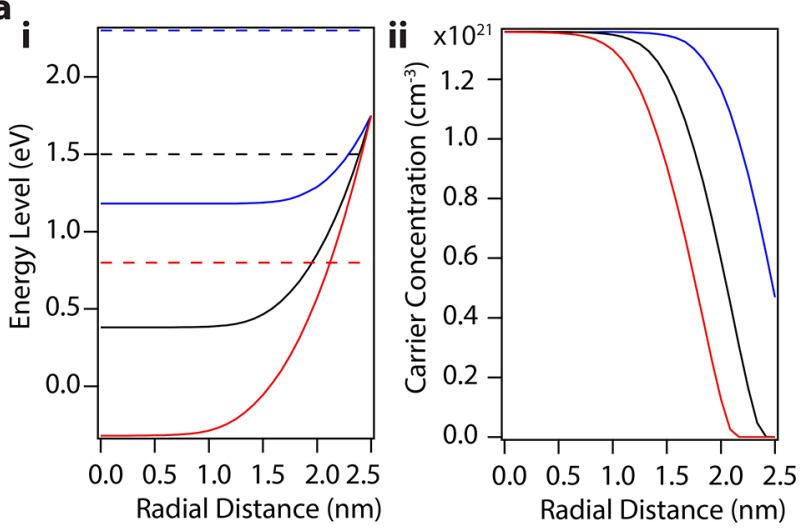

b

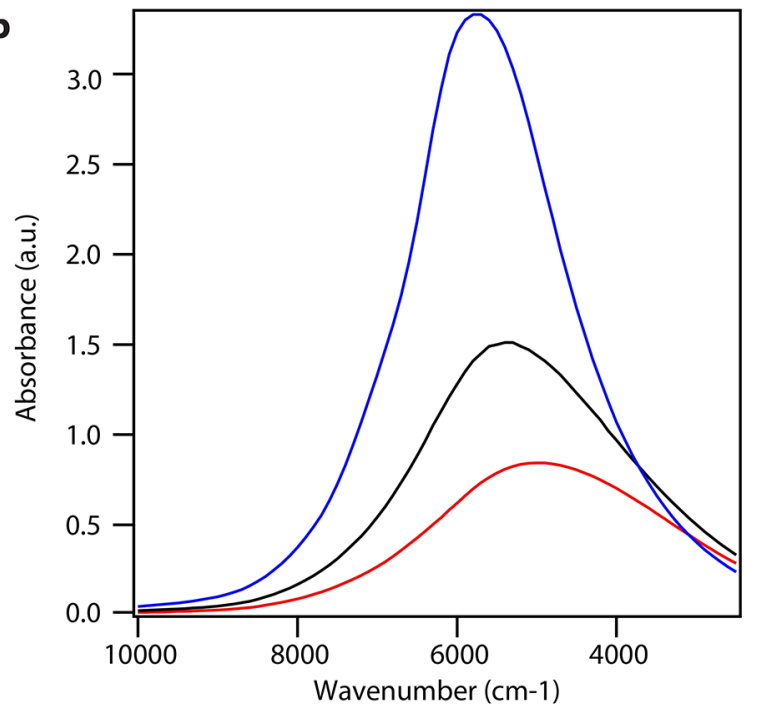

c

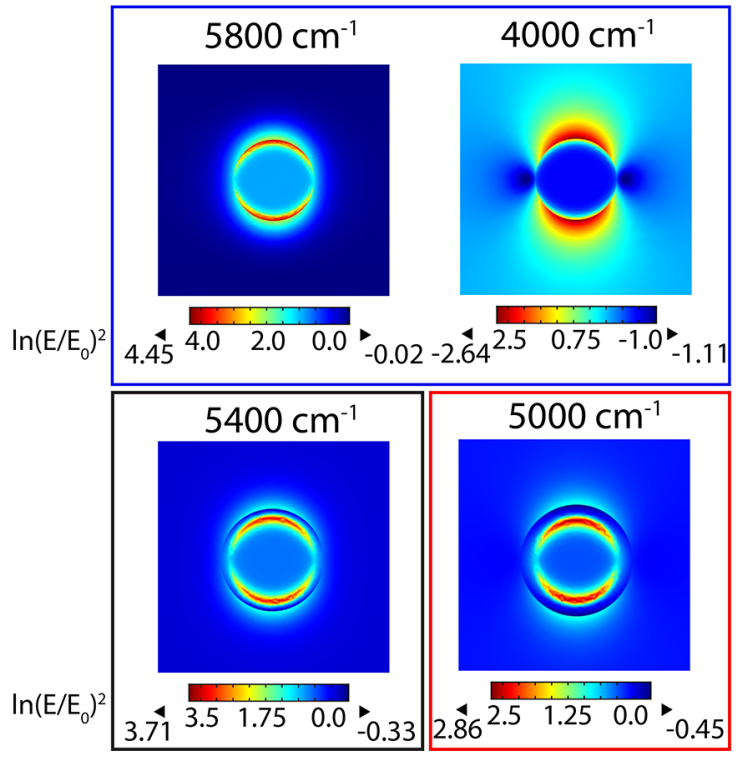

Figure 4. (a,i) Conduction energy band (solid lines) profile as a function of Fermi energy level (dashed lines), (ii) carrier concentration radial profile obtained from the solution to Poisson's equation. The depleted shell on the NC surface grows with increasingly oxidizing potential. (b) Simulated optical absorption spectra corresponding to the carrier concentration radial profiles in $(\mathrm{a}, \mathrm{ii})$. With increasing depletion width, LSPR spectra red-shift with substantial reduction in maximum LSPR absorption intensity. (c) Near-field maps simulated at the peak LSPR frequency corresponding to each of the LSPR spectra in (b). 
red-shifted peak accompanied by the decrease in LSPR intensity. With these simulated far-field optical properties and corresponding carrier distributions, we also simulated the nearfield optical properties inside and around the NCs. In Figure 4c we show the near-field enhancement intensity around the NCs for four different cases: the reduced case at two excitation wavelengths (red box), the neutral case (black), and the depleted case (blue), all excited at the maxima of the LSPR. We found that an increase in the depletion thickness leads to a decrease of the maximum near-field enhancement intensity when exciting at the peak LSPR frequencies of 5800, 5400, and $5000 \mathrm{~cm}^{-1}$, respectively. This loss in near-field enhancement is a result of the stronger contribution of the high refractive index shell. In general, the field enhancement is lower for thicker shells, such as those created under oxidative conditions (Figure 4c). It is important to note that the electric field enhancement is largely confined within the shell of higher dielectric, which might be disadvantageous for sensing applications. Similar field confinement inside a high dielectric shell has been shown earlier in silicon nanorod plasmonic systems. ${ }^{35,36}$ Nevertheless, the excitation of the near-field at higher wavelengths (or lower energies) with respect to the LSPR peak allows the field confinement to extend toward larger geometrical dimensions and into the surrounding dielectric medium. Though the maximum field enhancement is slightly lower in this case it provides a solution to circumvent the confinement of the field within the volume of the NC. Taken together our results highlight that a precise understanding of the plasmonic properties in relation to the extent of depletion is crucial for practical applications of doped semiconductor NCs in sensing, surface enhanced infrared absorption, or coupling to other optical processes such as excitons or phonons.

In conclusion, we have demonstrated reversible electrochemical charging and discharging of $\mathrm{Sn}: \mathrm{In}_{2} \mathrm{O}_{3}$ NCs dispersed in an electrolyte solution. The observed spectral shifts were rationalized using a core-shell optical model, by assuming an active NC core and a depleted shell. The modulation of the NC LSPR absorbance spectra upon electrochemical charging/ discharging was attributed to the change in the active core volume fraction. During reduction, the injection of carriers into the depletion region of the NC results in an increased core volume, which in turn leads to the substantial increase in absorption intensity and an only slight blue shift ascribable largely to decreased effectiveness of dielectric shielding. Similarly, during oxidation the decrease in absorption intensity along with LSPR redshift were correlated to the decrease in NC core volume fraction during oxidation as carriers are extracted from the outer core region only. In fact the depleted shell thickness at a specific potential matches well with the calculations performed on the NC solving Poisson's equations. The carrier density distribution within the NC volume was calculated and used for simulations of the near-field LSPR properties during the modulation process, suggesting that the thicker depletion shell reduces the maximum near-field enhancement around the NC but, moreover, that the nearfield was confined inside the high dielectric shell at peak LSPR frequency. Our results shed light on the electrochemical charging and discharging processes of doped NCs and highlight the importance of surface depletion on their plasmonic response.

\section{ASSOCIATED CONTENT}

\section{Supporting Information}

The Supporting Information is available free of charge on the ACS Publications website at DOI: 10.1021/acsphotonics.7b01587.

Detailed procedure of nanocrystal synthesis and electrochemical device, as well as mathematical information regarding spectra fitting and computation (PDF).

\section{AUTHOR INFORMATION}

\section{Corresponding Author}

*E-mail: milliron@che.utexas.edu.

ORCID

Ankit Agrawal: 0000-0001-7311-7873

Ilka Kriegel: 0000-0002-0221-3769

Evan L. Runnerstrom: 0000-0002-9054-708X

Anna Llordes: 0000-0003-4169-9156

Delia J. Milliron: 0000-0002-8737-451X

\section{Author Contributions}

"A.A. and I.K. contributed equally to this work. A.A. performed mathematical and computational analysis of experimental results. I.K. performed all electrochemical experiments in collaboration with A.L. E.L.R performed nanocrystal synthesis and characterization. All authors contributed to writing of the final manuscript.

\section{Notes}

The authors declare no competing financial interest.

\section{ACKNOWLEDGMENTS}

A.A., E.L.R., and A.L. were supported by the National Science Foundation (NSF, CHE-1609656) and the Welch Foundation (F-1848). I.K. and F.S. received funding from the European Union's Horizon 2020 research and innovation programme (MOPTOPus) under the Marie Skłodowska-Curie Grant Agreement No. (705444), as well as (SONAR) Grant Agreement No. (734690).

\section{REFERENCES}

(1) Agrawal, A.; Johns, R. W.; Milliron, D. Control of Localized Surface Plasmon Resonances in Metal Oxide Nanocrystals. Annu. Rev. Mater. Res. 2017, 47 (1), 1-31.

(2) Kriegel, I.; Scotognella, F.; Manna, L. Plasmonic Doped Semiconductor Nanocrystals: Properties, Fabrication, Applications and Perspectives. Phys. Rep. 2017, 674, 1-52.

(3) Diroll, B. T.; Gordon, T. R; Gaulding, E. A.; Klein, D. R.; Paik, T.; Yun, H. J.; Goodwin, E. D.; Damodhar, D.; Kagan, C. R.; Murray, C. B. Synthesis of N-Type Plasmonic Oxide Nanocrystals and the Optical and Electrical Characterization of Their Transparent Conducting Films. Chem. Mater. 2014, 26 (15), 4579-4588.

(4) Lounis, S. D.; Runnerstrom, E. L.; Llordés, A.; Milliron, D. J. Defect Chemistry and Plasmon Physics of Colloidal Metal Oxide Nanocrystals. J. Phys. Chem. Lett. 2014, 5 (9), 1564-1574.

(5) Agrawal, A.; Kriegel, I.; Milliron, D. J. Shape-Dependent Field Enhancement and Plasmon Resonance of Oxide Nanocrystals. J. Phys. Chem. C 2015, 119 (11), 6227-6238.

(6) Wang, Y.; Runnerstrom, E. L.; Milliron, D. J. Switchable Materials for Smart Windows. Annu. Rev. Chem. Biomol. Eng. 2016, 7 (1), $283-$ 304.

(7) Schimpf, A. M.; Knowles, K. E.; Carroll, G. M.; Gamelin, D. R. Electronic Doping and Redox-Potential Tuning in Colloidal Semiconductor Nanocrystals. Acc. Chem. Res. 2015, 48 (7), 1929-1937.

(8) Mendelsberg, R. J.; McBride, P. M.; Duong, J. T.; Bailey, M. J.; Llordes, A.; Milliron, D. J.; Helms, B. A. Dispersible Plasmonic Doped 
Metal Oxide Nanocrystal Sensors That Optically Track Redox Reactions in Aqueous Media with Single-Electron Sensitivity. Adv. Opt. Mater. 2015, 3 (9), 1293-1300.

(9) Garcia, G.; Buonsanti, R.; Runnerstrom, E. L.; Mendelsberg, R. J.; Llordes, A.; Anders, A.; Richardson, T. J.; Milliron, D. J. Dynamically Modulating the Surface Plasmon Resonance of Doped Semiconductor Nanocrystals. Nano Lett. 2011, 11 (10), 4415-4420.

(10) Kim, J.; Ong, G. K.; Wang, Y.; LeBlanc, G.; Williams, T. E.; Mattox, T. M.; Helms, B. A.; Milliron, D. J. Nanocomposite Architecture for Rapid, Spectrally-Selective Electrochromic Modulation of Solar Transmittance. Nano Lett. 2015, 15 (8), 5574-5579.

(11) Heo, S.; Kim, J.; Ong, G. K.; Milliron, D. J. Template-Free Mesoporous Electrochromic Films on Flexible Substrates from Tungsten Oxide Nanorods. Nano Lett. 2017, 17 (9), 5756-5761.

(12) zum Felde, U.; Haase, M.; Weller, H. Electrochromism of Highly Doped Nanocrystalline $\mathrm{SnO}_{2}:$ Sb. J. Phys. Chem. B 2000, 104 (40), 9388-9395.

(13) Zandi, O.; Agrawal, A.; Shearer, A. B.; Gilbert, L. C.; Dahlman, C. J.; Staller, C. M.; Milliron, D. J. Impacts of Surface Depletion on the Plasmonic Properties of Doped Semiconductor Nanocrystals. ArXiv170907136 Cond-Mat Physicsphysics 2017, na.

(14) Feigenbaum, E.; Diest, K.; Atwater, H. A. Unity-Order Index Change in Transparent Conducting Oxides at Visible Frequencies. Nano Lett. 2010, 10 (6), 2111-2116.

(15) Müller, J.; Sönnichsen, C.; von Poschinger, H.; von Plessen, G.; Klar, T. A.; Feldmann, J. Electrically Controlled Light Scattering with Single Metal Nanoparticles. Appl. Phys. Lett. 2002, 81 (1), 171-173.

(16) Byers, C. P.; Zhang, H.; Swearer, D. F.; Yorulmaz, M.; Hoener, B. S.; Huang, D.; Hoggard, A.; Chang, W.-S.; Mulvaney, P.; Ringe, E.; et al. From Tunable Core-Shell Nanoparticles to Plasmonic Drawbridges: Active Control of Nanoparticle Optical Properties. Sci. Adv. 2015, 1 (11), e1500988.

(17) Chirea, M.; Collins, S. S. E.; Wei, X.; Mulvaney, P. Spectroelectrochemistry of Silver Deposition on Single Gold Nanocrystals. J. Phys. Chem. Lett. 2014, 5 (24), 4331-4335.

(18) Novo, C.; Funston, A. M.; Gooding, A. K.; Mulvaney, P. Electrochemical Charging of Single Gold Nanorods. J. Am. Chem. Soc. 2009, 131 (41), 14664-14666.

(19) Dondapati, S. K.; Ludemann, M.; Müller, R.; Schwieger, S.; Schwemer, A.; Händel, B.; Kwiatkowski, D.; Djiango, M.; Runge, E.; Klar, T. A. Voltage-Induced Adsorbate Damping of Single Gold Nanorod Plasmons in Aqueous Solution. Nano Lett. 2012, 12 (3), $1247-1252$.

(20) Mulvaney, P. Surface Plasmon Spectroscopy of Nanosized Metal Particles. Langmuir 1996, 12 (3), 788-800.

(21) Mulvaney, P.; Pérez-Juste, J.; Giersig, M.; Liz-Marzán, L. M.; Pecharromán, C. Drastic Surface Plasmon Mode Shifts in Gold Nanorods Due to Electron Charging. Plasmonics 2006, 1 (1), 61-66.

(22) Ung, T.; Giersig, M.; Dunstan, D.; Mulvaney, P. Spectroelectrochemistry of Colloidal Silver. Langmuir 1997, 13 (6), 1773-1782.

(23) Zhang, X.; Hicks, E. M.; Zhao, J.; Schatz, G. C.; Van Duyne, R. P. Electrochemical Tuning of Silver Nanoparticles Fabricated by Nanosphere Lithography. Nano Lett. 2005, 5 (7), 1503-1507.

(24) Guyot-Sionnest, P. Charging Colloidal Quantum Dots by Electrochemistry. Microchim. Microchim. Acta 2008, 160 (3), 309314.

(25) Scanlon, M. D.; Peljo, P.; Méndez, M. A.; Smirnov, E.; Girault, H. H. Charging and Discharging at the Nanoscale: Fermi Level Equilibration of Metallic Nanoparticles. Chem. Sci. 2015, 6 (5), 27052720.

(26) Choi, S.-I.; Nam, K. M.; Park, B. K.; Seo, W. S.; Park, J. T. Preparation and Optical Properties of Colloidal, Monodisperse, and Highly Crystalline ITO. Chem. Mater. 2008, 20 (8), 2609-2611.

(27) Kanehara, M.; Koike, H.; Yoshinaga, T.; Teranishi, T. Indium Tin Oxide Nanoparticles with Compositionally Tunable Surface Plasmon Resonance Frequencies in the Near-IR Region. J. Am. Chem. Soc. 2009, 131 (49), 17736-17737.

(28) Runnerstrom, E. L.; Llordés, A.; Lounis, S. D.; Milliron, D. J. Nanostructured Electrochromic Smart Windows: Traditional Materials and NIR-Selective Plasmonic Nanocrystals. Chem. Commun. 2014, 50 (73), 10555-10572.

(29) Krause, K. J.; Mathwig, K.; Wolfrum, B.; Lemay, S. G. Brownian Motion in Electrochemical Nanodevices. Eur. Phys. J.: Spec. Top. 2014, 223 (14), 3165-3178.

(30) Robbs, P. H.; Rees, N. V. Nanoparticle Electrochemistry. Phys. Chem. Chem. Phys. 2016, 18 (36), 24812-24819.

(31) Schimpf, A. M.; Gunthardt, C. E.; Rinehart, J. D.; Mayer, J. M.; Gamelin, D. R. Controlling Carrier Densities in Photochemically Reduced Colloidal ZnO Nanocrystals: Size Dependence and Role of the Hole Quencher. J. Am. Chem. Soc. 2013, 135 (44), 16569-16577.

(32) Hoener, B. S.; Byers, C. P.; Heiderscheit, T. S.; Indrasekara, A. S. D. S.; Hoggard, A.; Chang, W.-S.; Link, S.; Landes, C. F. Spectroelectrochemistry of Halide Anion Adsorption and Dissolution of Single Gold Nanorods. J. Phys. Chem. C 2016, 120 (37), 2060420612.

(33) Brown, A. M.; Sheldon, M. T.; Atwater, H. A. Electrochemical Tuning of the Dielectric Function of Au Nanoparticles. ACS Photonics 2015, 2 (4), 459-464.

(34) Seiwatz, R.; Green, M. Space Charge Calculations for Semiconductors. J. Appl. Phys. 1958, 29 (7), 1034-1040.

(35) Boyuk, D. S.; Chou, L.-W.; Filler, M. A. Strong Near-Field Coupling of Plasmonic Resonators Embedded in Si Nanowires. ACS Photonics 2016, 3, 184-189.

(36) Chou, L.-W.; Near, R. D.; Boyuk, D. S.; Filler, M. A. Influence of Dielectric Anisotropy on the Absorption Properties of Localized Surface Plasmon Resonances Embedded in Si Nanowires. J. Phys. Chem. C 2014, 118 (10), 5494-5500. 